\title{
Il Sistema Bancario di Fronte ai Problemi Economici
}

By

\author{
${ }^{1}$ Dr. Anilda Bozdo, ${ }^{2}$ Dr. Mimoza Koka
}

\begin{abstract}
The deteriorating reasons of banking portfolio loans and some necessary measures for the improvement of this situation will be main topics of this article. The corruption in the banking system, is a phenomenon that has passed unobserved, but for our opinion is very often. Non professional loan officers, who lack the essential knowledge of identifying the clients, are common among the Banks of Albania; The new banking system, appears with the old problems of expansion of loan portfolio during 2005-2007, without the bank capacity to manage them and that for sure will be accompanied with influx of many clients with problems. The objective reason is also economic situation of our country and our region that for sure will reflect in quality of loans portfolio;

The measures that are important to take are: a reform in banking system for what it concerned structure management in charge of loans giving. The loan officers reward should be connected with quality of portfolio management. Bank of Albania should enforce banks to spend more for training the loan officer. It may organize itself the training courses by raising a training center. It should control on-site banks more frequently. Should pay attention on giving new loans, which will improve gradually the quality of loan portfolio. Despite the situation must not restraint the loan process in bank.
\end{abstract}

Keywords : Nonperforming loans, loan officer; corruption; financial crisis.

\section{Introduzione}

La crisi finanziaria mondiale e una crisi che e stata ampiamente discussa nella carta stampata da parte degli economisti di diverse scuole, ma che tutti quanti si uniscono nell'idea che questa crisi ebbe la sua origine nei "sub prime mortgage meltdown" negli Stati Uniti d'America nell'anno 2006-2007. Abbiamo una serie di economisti che hanno studiato la crisi e sulla quale, hanno diversi orientamenti come Gary Becker, Richard Posner, Ana Schwartz, Elinor Ostrom, Mario Draghi(Draghi, 2009), Jean-Claude Trichet (Trichet, 2009) Paul Krugman, ma che tutti sono d'accordo con l'affermazione di B.S. Bernake (Bernanke and Gertler, 1995) del ruolo della politica economica nel sostegno del credito, per il quale, il credito da parte delle banche e l'unico modo per aproviggionare l'economia di liquidità. 
Questo ha fatto si che la Banca d'Albania (Fullani 2010) giocasse un ruolo fondamentale nella promozione del sistema bancario nel dare nuovo credito all'economia, usando come strumento di leva il tasso d'interesse, o anche altre misure di natura amministrativa.

Nel fra tempo la Banca Centrale Albanese stimo che la situazione e la performance del sistema bancario e quello finanziario sarebbero rimaste stabili. I dati sulla liquidità e la capitalizzazione erano a un livello sufficientemente buono.

\section{Una Visuale Generale del Sistema Bancario}

In Albania operano attualmente sedici banche, la dove Raiffesen Bank, Intesa San Paolo Bank e la Banka Kombëtare Tregtare avevano il 57.6\% del totale degli attivi del sistema bancario nel Dicembre 2012.

Grafico 1: La struttura dell'attivo nel sistema bancario, dicembre 2012

\begin{tabular}{|c|c|}
\hline La struttura dell' attivo nel sis:eme banzaric, dicembre 2012 & 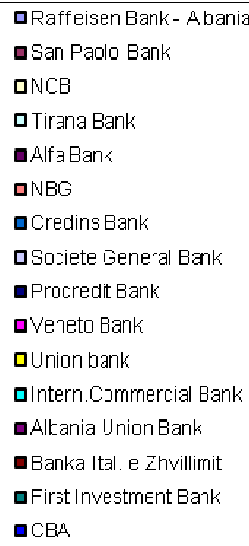 \\
\hline
\end{tabular}

Quali erano le caratteristiche principali della situazione finanziaria delle banche banche in Albania:

a) In relazione al portafoglio crediti: possiamo dire che quasi tutte le banche del sistema hanno avuto problemi. Questo anche per timore che le aumentate difficoltà per le imprese dovute alla crisi potessero avere ripercussioni nel estinzione dei loro debiti nei confronti delle banche. Le banche rallentarono il loro processo creditizio facendo si che diminuisse anche i ritmo di crescita del credito. Dopo l'anno 2012, le banche non solo fermarono i ritmi di crescita del credito, ma si ebbe una diminuzione. Nel fra tempo il rapporto credito/depositi diminuì, (dal 64.80\% nel Dicembre 2009 al 58.27\% del Dicembre 2012), aiutando cosi nel miglioramento delle problematiche di liquidità delle banche, ma d'altro canto nell'abbassamento del reddito da interesse questo a causa dello spostamento dei capitali in attivi meno redditizi, come il deposito in altre banche oppure nei titoli di stato (buono del tesoro). 


\section{II trend dei prestiti 2006-2012}

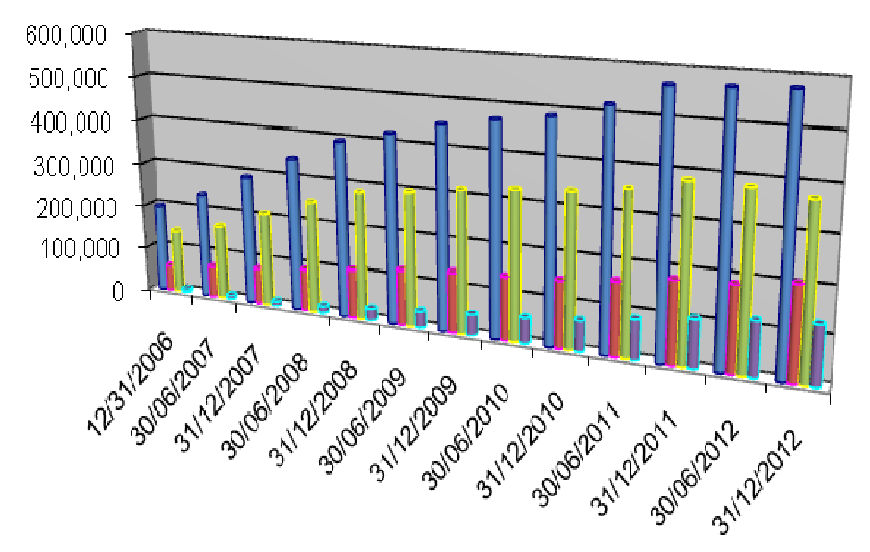

-

Nel fra tempo il rapporto dei crediti con problemi sul totale del credito si innalzo in maniera galoppante, diventando cosi una seria preoccupazione per le banche e per l'economia.

\section{Grafico 3: Prestiti/Depositi rapporto ${ }^{2}$}

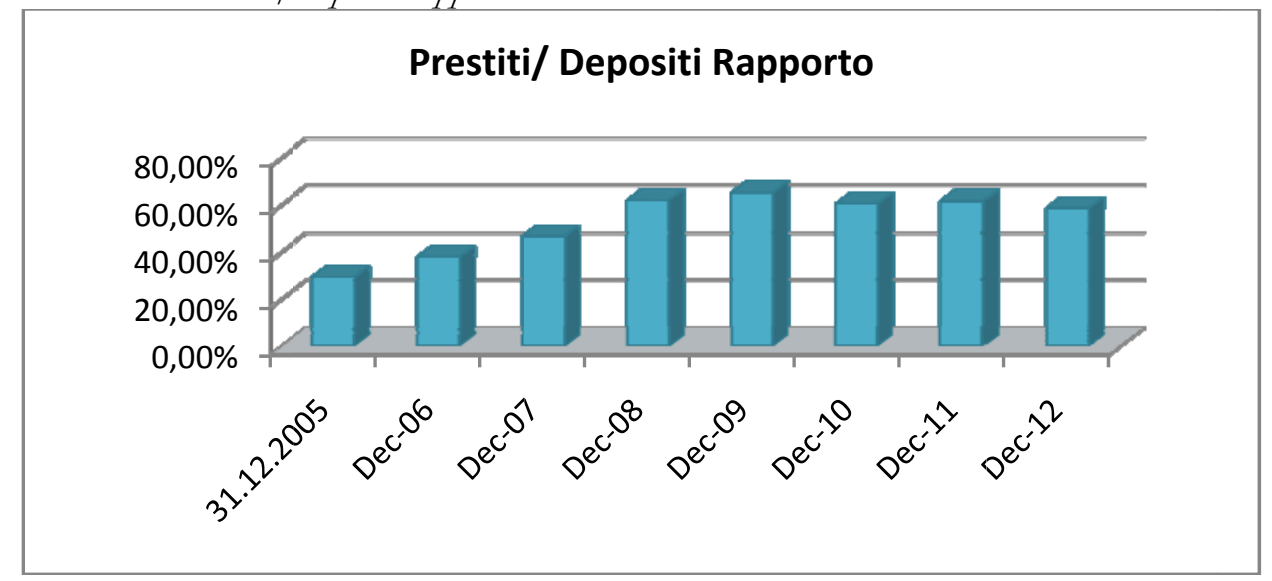

b) Sui depositi. Un fenomeno positivo, perche a parte la fine del 2008 e l'inizio del 2009, i depositi sono stati caratterizzati da una crescita. Se vediamo il periodo Dicembre 2009 - Dicembre 2012 i depositi sono cresciuti del 42.84\%. Questo per una serie di ragioni:

- $\quad$ La paura del consumatore di consumare per colpa della crisi

\footnotetext{
${ }^{1}$ Bank of Albania, Annual Supervision Report 2012

2 Sempre li
} 
- L'affluire di depositi da parte degli emigranti in Grecia, per colpa della paura di un possibile fallimento delle banche Greche. Questo si capisce anche dal fatto del cambiamento della struttura dei depositi, dove prima il maggior peso dei depositi era costituito da Lek e adesso quello in valuta estera, indipendentemente dal fatto che i tassi di interesse sulla moneta nazionale erano più alti di quelli per le valute estere.

- L'impatto in ritardo delle conseguenze della crisi in Albania, avendo ancora una crescita del 3-4\% del PIL annuo. Solo dopo il 2012 si può dire che questa crisi si sta sentendo potentemente nell'economia Albanese

c) Grafico 4: Il trend dei Depositi 2006-2012

\section{Il trend dei prestiti 2006-2012}

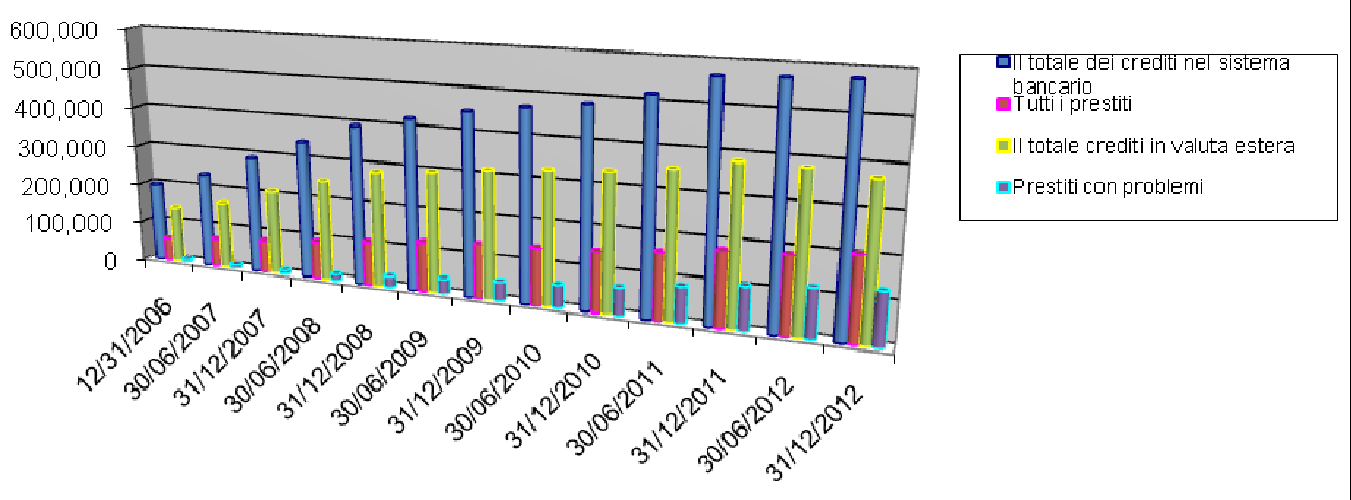

d) Sul Capitale azionario. Il totale degli attivi fino a dicembre 2013 e cresciuto del $34.37 \%$ paragonato al 31 dicembre 2009. questo come causa della crescita dei depositi e del capitale pagato dalle banche. Il capitale azionari e cresciuto del $20.03 \%$. questo anche perl'impatto positivo delle ricchieste fatte dalla Banca d'Albania per non far distribuire i dividenti per l'anno 2009, par fare aumentare cosi il livello di liquidita nel sistema bancario. In questa maniera tutto il guadagnio realizzato nel 2009 si e aggiunto al capitale delle banche. 
Grafico 5:Il trend degli attivi e del capitale ${ }^{3}$

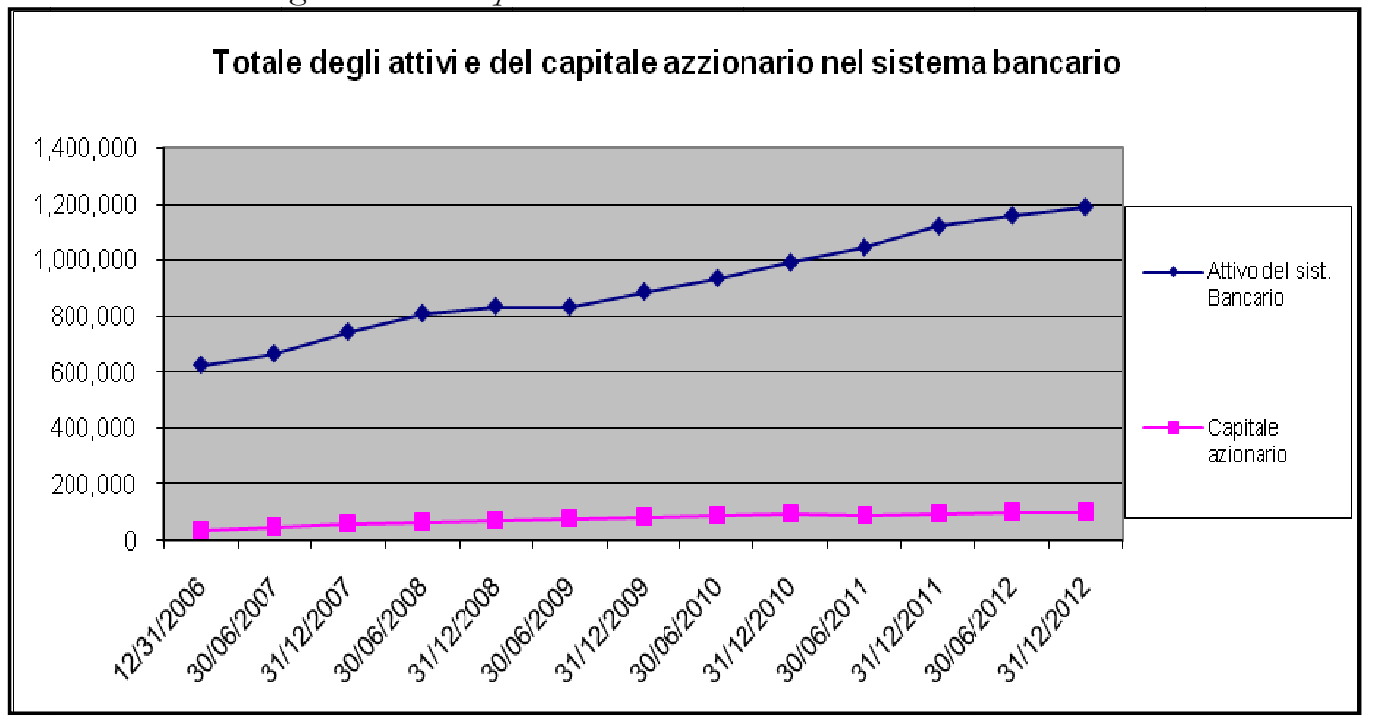

La Banca d'Albania ha chiesto alle banche di aumentare il livello del capitale versato da 700 milioni di LEK che era ad 1 miliardo di LEK. Tutti gli azzionisti delle banche si sono dimostrati disponibili ad alzare questo livello, e quest ha fatto si che si alzasse il livello di capitalizzazione delle banche. Tutte le banche non avevano problemi con la norma del adeguadezza del capitale, restano sopra il livello minimale deciso dalla Banca d'Albania del $12 \%$.

Grafico 6: La norma dell'adeguatezza del capitale netto2012

la norma dell'adeguatezza del capitale netto

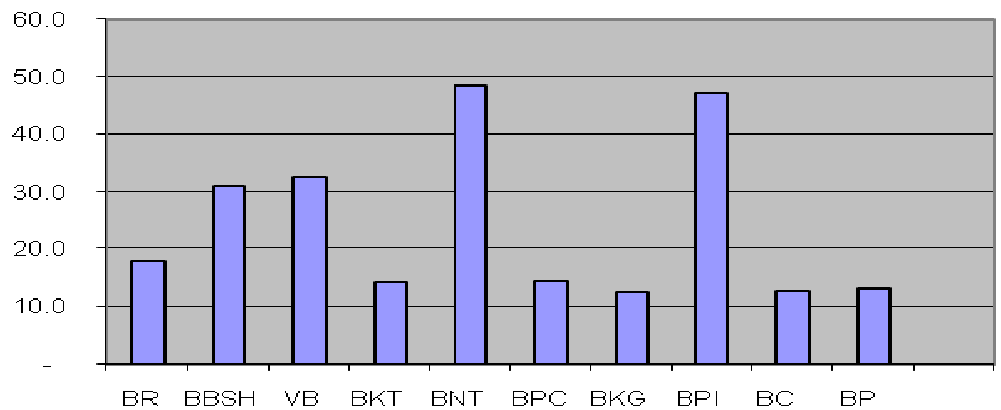

\section{Problema del processo creditizio in Albania}

\footnotetext{
${ }^{3}$ Sempre li
} 
Il credito in Albania ebbe un palpabile peggioramento negli ultimi anni. Quali furono le cause del peggioramento di questo rapporto, che e un indicatore molto importante per giudicare la stabilita del sistema bancario?

a) La causa principale e la corruzione che esiste nel sistema bancario, fenomeno al quale non si sta dando la necessaria importanza, ma a seconda del mio avviso e un fenomeno assai diffuso. Questo ha fatto si che si dessero crediti, i quali non soddisfavano tutti $i$ requisiti per un loro normale andamento, e creando cosi difficoltà ai clienti sani di ottenere il credito i quali non lubrificano gli ingranaggi... Il risultato e che il sistema sostiene il peso di crediti dati da persone corrotte.

b) Il livello professionale non adatto da parte degli operatori del credito, i quali si accontentavano di adempiere in maniera formale alle richieste imposte dalla Banca d'Albania, prevalentemente sul collaterale e sul livello del reddito,non sono in grado di capire e conoscere i clienti. Questi clienti non hanno la prospettiva delle loro imprese, nonostante abbiano un collaterale sufficiente ad ottenere un credito. Nel frattempo ci sono anche dei casi in cui questi clienti possono avere una situazione economica difficile, ma che lo possono nascondere lavorando con doppio bilancio, quello per il fisco e quello "reale". Le Banche, essendo che si assottiglierebbe lo spazio per dare il credito se si basassero solo sul bilancio per il fisco, danno il credito basandosi anche sul bilancio messo a disposizione da parte delle imprese come reale. Simili bilanci aumentano di molto il rischio di dare il credito ai clienti con problemi, i quali possono mostrare dati gonfiati sui loro ricavi. In questo caso, gli operatori del credito debbono essere professionalmente capaci e abili a capire le cifre mostrategli,le quali possono avere tra di loro discrepanze e contraddizioni, se sono state compilate in maniera non reale, come anche dal controllo sul campo da parte di questi ultimi, che siano capaci di saper cosa devono controllare ed analizzare i dati mostratigli delle imprese per attestare la loro veridicità. Questo e un rischio abbastanza evidente, perche l'economia informale a mio avviso ha un peso non trascurabile nell'economia.

Grafico 7: La struttura dei prestiti con problemi a seconda dei settori economici

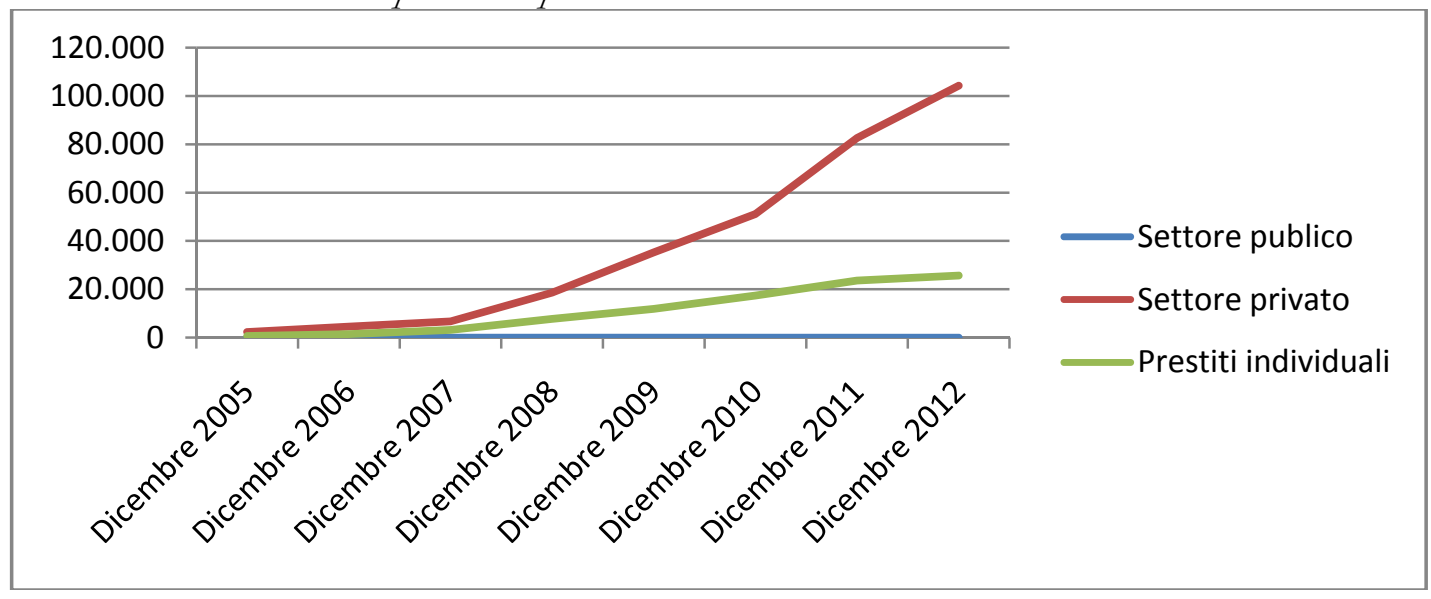


c) Il sistema bancario e relativamente giovane nel dare il credito, se pensiamo che i crediti ipotecari per le case sono crediti 15-20 anni( che occupano più o meno la meta del portafoglio dei crediti di una banca). I crediti, si pensa teoricamente, cominciano a capirsi se hanno problemi all'incirca dopo 1/3 della loro durata, perche all' inizio le rate vengono pagate attraverso lo stesso credito preso, una parte viene usata per le necessita delle imprese e una parte per far fronte al pagamento dello stesso prestito. Lo stesso secondo il regolamento della Banca d'Albania un prestito con problemi viene chiamato tale quando e passato almeno un periodo di tre mesi senza che le rate di quest'ultimo vengano pagate. Cosi che molte banche all'inizio possono avere portafogli in buono stato, nonostante che il livello dei loro clienti non sia altrettanto buono. Nel frattempo anche il nostro sistema bancario, nonostante che ormai e da 15 anni che e iniziato il processo dei prestiti, solamente negli ultimi anni i prestiti hanno cominciato ad avere un peso importante negli totali degli attivi di una banca ( nel Dicembre 2010 49.10\% dei totali degli attivi, nel dicembre 2011 al 50.55\% degli attivi e nel dicembre 2012 48.63\%), aumentando in maniera rilevante anche il rischi derivante da essi. Nonostante ciò possiamo dire che il peso che questi prestiti occupano non e abbastanza grande, se no il rischio rappresentato dal rapporto prestiti con problemi/ totale prestiti, nel Dicembre 2012 22.49\%, Dicembre 2011 al 18.77\%, Dicembre 2009 al 10.48\% e nel Dicembre 2008 al 6.66\%, dovrebbe essere molto serio. Questo rapporto e aumentato di tre volte in quattro anni(Dicembre 2008-Dicembre 2012), che sta a significare di un suo forte incremento.

Grafico 8: La struttura dei prestiti con problemi a seconda della scadenza.

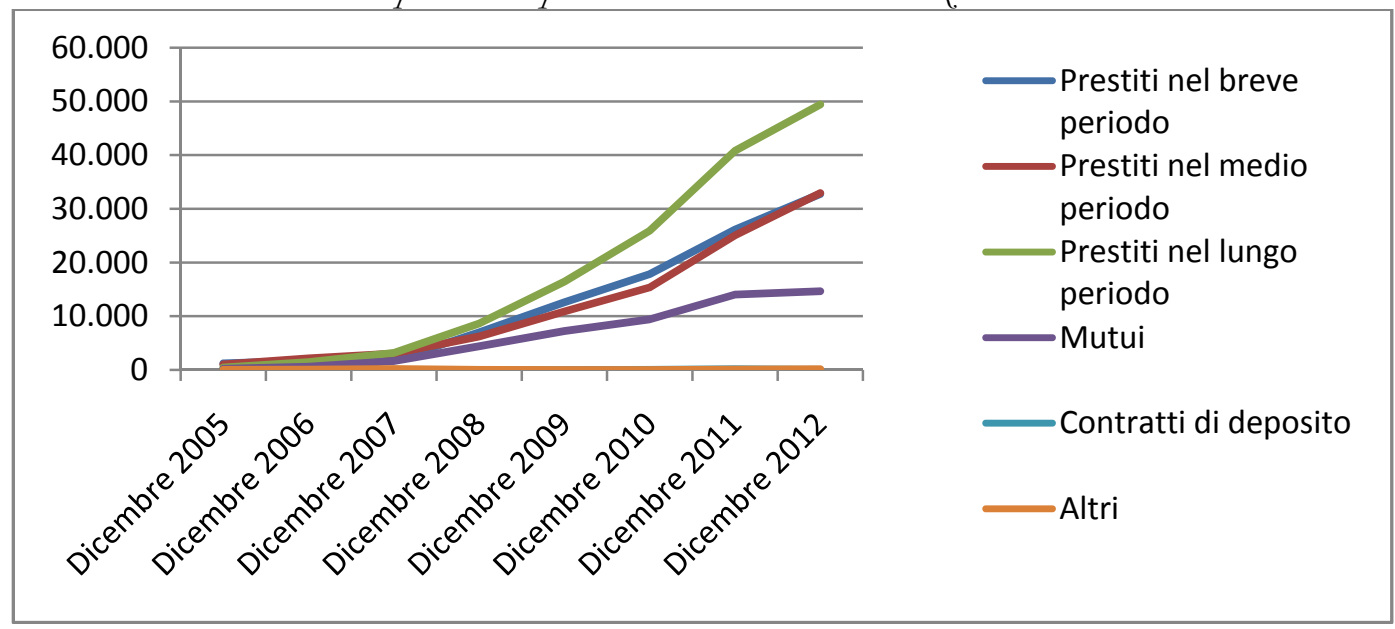

d) La ragione obbiettiva e anche la situazione economica del nostro paese ma ance della regione circostante, che senz'altro si riflette nella qualità dei portafogli prestiti. Questo e il risultato delle difficoltà che gli individui hanno nel pagare le rate dei prestiti, se questi ultimi anno perso la fonte da guadagno ( il reddito da salario), oppure le imprese come causa che una parte di loro sono fallite. Non possiamo anche dimenticare che una buona parte dei prestiti ipotecari-mutui sono stati presi dagli emigranti, i quali 
possono aver perso il posto di lavoro nei paesi vicini come Italia e Grecia, perche la classe più colpita nei periodi di crisi sono gli emigranti. I prestiti con problemi per gli individui sono cresciuti durante il periodo dicembre 2009/ dicembre 2012 con il 116\% avendo il $19.74 \%$ del peso sul totale dei prestiti con problemi in questo periodo.

e) Una ragione e anche l'espansione dell'offerta dei prestiti di due anni fa, l'accelerazione da parte delle banche nel dare prestiti, senz'altro sarebbe stata accompagnata da una penetrazione da parte di più clienti con problemi. Nonostante una frenata della crescita dei prestiti in questi ultimi anni( dal 35.6\% dicembre 2007/2008 al $13.1 \%$ dicembre $2008 / 2009$ al $9.1 \%$ dicembre $2009 / 2010$ fino al $2.05 \%$ del dicembre 2011/2012). La grande crescita del 2008, essendo che esiste un gap nel legame del tempo necessario affinché si presentino le conseguenze, si rifletterà oggi con la crescita dei prestiti con problemi.

Pensiamo che la situazione sia seria e che la Banca d'Albania debba comportarsi in maniera molto cauta in questa situazione.

Non e una maniera per difendersi il rallentamento nel dare nuovi prestiti dato che ormai i collaterali sono al livello di $140-150 \%$ del totale del prestito chiesto. Spesso questa richiesta di questi collaterali ha anche una conseguenza negativa nell'economia, penalizzando impresse di successo, con chiare prospettive di crescita, ma che non hanno proprietà immobiliari per sostenere le loro richieste di prestito.

Quali potrebbero essere le misure da intraprendere?

Penso che ce spazio per una riforma nel sistema bancario, nelle strutture adibite ad elargire il prestito.

a) Il salario ed i corrispettivi bonus degli operatori del credito debbono essere sempre più spesso collegati alla qualità del portafoglio da loro gestito. La decisione sul dare o meno un prestito deve essere decentralizzata quando quest'ultimo e di piccola quantità, questa decisione non sempre deve essere collettiva cosi che ance la responsabilità non sarà collettiva. Per piccole somme la decisione dovrà essere unicamente degli agenti dei prestiti, e la responsabilità completamente loro. Alcune banche nel nostro sistema appiccano questo metodo come ProCredit Bank e hanno avuto risultati molto buoni nel processo dei prestiti. Banche più conservatrici anche nei bassi livelli del processo del prestito, la decisione veniva preso da un comitato, i risultati sono stati più deludenti. Dalla mia esperienza formativa negli USA, questa era una forma largamente praticata la giu.

b) La Banca d'Albania deve chiedere che le banche spendano di più in corsi di formazione per i loro agenti del credito facendo anche un loro allontanamento dal lavoro. Negli USA le banche ogni anno mandavano i loro agenti in corsi di formazione di 15-30 giorni, come anche per le metodiche del processo del prestito, cosi come anche per quello della conoscenza legislazione. I nostri agenti sono cosi carichi dei loro fascicoli che spesso loro non hanno il tempo di formarsi individualmente, ma neanche di leggere le regolamentazione promulgata dalla Banca d'Albania sul processo dei prestiti bancari.

c) La stessa Banca d'Albania dovrebbe tenere dei corsi di formazione per gli agenti del credito, creando un centro di formazione. Questo centro funzionava tempo fa dando lezioni per i dipendenti delle banche non solo per il processo del prestito ma anche per molti altri aspetti sul sistema bancario. 
d) La Banca d'Albania dovrebbe velocizzare i controlli tematici on-site sul prestito, specialmente quest'anno, per diagnosticare più precisamente i problemi per ogni banca in maniera individuale, penalizzando individualmente i colpevoli.

e) porre molta attenzione quando si danno i nuovi prestiti, le quali s fossero prestiti genuini, porterebbero in un graduale miglioramento della qualità dei portafogli prestiti. Quindi nonostante la situazione, non si dovrebbe fermare il processo dei prestiti da parte delle banche.

f) Le banche dovranno gestire con molta attenzione i loro portafoglio prestiti. Dovranno essere svolti controlli da parte degli agenti del credito presso le imprese almeno una volta l'anno. Questi controlli dorranno servire per conoscere prima la situazione, cosi che le banche potranno prendere misure precedentemente affinché questi prestiti possano essere restituiti. Questo attraverso la ristrutturazione dei prestiti, la dove questo possa dare un risultato, per non fare fallire un impresa, o la dove non ci sia più speranza per il ritorno del prestito, facendo l'aproviginamento necessario, e giocando di anticipo sulle procedure del pignoramento del collaterale.

g) devono essere migliorate e implementate i manuali del prestito da parte delle banche, in modo che questi manuali siano un documento molto esaustivo e quindi una guida per gli agenti del credito, cosi da prendere in considerazione e comprendere in essi anche i nuovi prodotti che le banche stanno immettendo nel mercato, e tenere conto anche delle situazioni che il nostro paese sta passando. Queste procedure al completo, preverranno il disborso di prestiti con problemi.

h) Le banche devono essere molto caute ed attente nella diversificazione del portafoglio crediti, seguendo con attenzione che non ci siano concentrazioni in settori particolari dell'economia come ad esempio l'edilizia. Se cominceranno a vedersi problemi sotto questo punto di vista, loro prenderanno la forma di una valanga, perche la banca ha permesso grandi concentrazioni non solo nel settore dell'edilizia ma anche nelle imprese della filiera collegate all'edilizia. Le banche devono cercare di separare in settori più diversificati possibili la loro attività creditizia.

i) Per gli individui che prendono casa, le banche devono essere molto rigorose a giudicare nella maniera in cui essi sono stati stabili o meno nel luogo di lavoro. Per i lavoratori a tempo, oppure con un lavoro non stabile, le banche devono essere più conservatrici nel erogare il credito.

Noi pensiamo,che queste sono le misure indispensabili da intraprendere da parte delle banche, ma la cosa più importante e il trattamento della situazione da parte delle banche con la massima seriosità, perche questo rapporto dei prestiti con problemi/ soul totale prestiti, e molto grave. I problemi non si risolvono frenando il processo dell'attività creditizia perche questo sarebbe un corto circuito. Se l'economia non si sviluppa e non cresce neanche i credito non sarà qualitativo. 


\section{I Problemi del Collaterale} collaterale.

Un altro problema che esiste nell'economia albanese e il problema del Secondo il governatore della Banca d'Albania ${ }^{4}$, le banche di secondo livello devono cominciare le procedure di pignoramento del collaterale, cosa che aiuterebbe nel miglioramento del rapporto dei prestiti con problemi/ soul totale prestiti.

In principio questo e esatto, perche il pignoramento del collaterale vorrebbe dire la restituzione di una parte del debito da parte dei clienti verso la banca, cosa che aiuterebbe nella diminuzione di questo rapporto.

Nonostante ciò noi pensiamo che non sia una cosa cosi semplice perche da essa derivano una serie di problemi nel nostro sistema bancario.

Quali sono questi problemi?

Primo, non tutti i prestiti sono coperti da un collaterale, che e una proprietà immobiliare come una casa, edificio o un terreno. Una parte dei prestiti, come sono i prestiti dei consumatori oppure fidi bancari, non è garantiti da un collaterale, in questo caso il collaterale non ha nessun effetto nel minimizzare i prestiti con problemi dati dal sistema bancario. Non dimentichiamoci che nelle condizioni della crisi, che si sta facendo sentire in Albania, anche l'economia che si sta dirigendo verso una situazione di stagnazione, e il PIL ha avuto una diminuzione considerevole. L'aumentare del nemeo dei disoccupati, fenomeno acetato dall'ISTAT ${ }^{5}$, vorrebbe dire che una buona parte delle persone che hanno preso fidi bancari basati sulla quantità di salario che ha avuto, perdendo il posto di lavoro, non ha più la capacita di fare fronte alla restituzione delle somme prestategli. Questo sarebbe una perdita netta da parte delle banche, perche esse non hanno avuto altri mezzi per coprire questi fidi se non la paga che essi prendevano. La stessa cosa vale anche per i prestiti al consumatore.

D’altra parte perché questi prestiti hanno un tasso di rischio più allevato per le banche, hanno tassi d'interesse più alti, questi tassi variano dal 16-22\% e in alcuni casi anche di più secondo la banca. Questo ha influito che questi prestiti abbiano la predisposizione maggiore per avere dei problemi. Quello che si nota nel nostro sistema bancario, che potremmo dire che e un fenomeno molto negativo, e il fatto che nonostante la Banca d'Albania ha portato al minimo il tasso base d'interesse (4.25\%). Il livello dei tassi d'interesse nel mercato bancario non e sceso di pari passo a quelli della Banca d'Albania. Questo perche le banche si sentono minacciate dai prestiti con problemi. Con i tassi d'interesse alti per i nuovi prestiti le banche tentano di coprire le perdite derivanti dai prestiti con problemi. Questo e un corto circuito perche proprio questi tassi alti possono essere una delle ragioni, oltre a quelli macroeconomici, dell'aumento dei prestiti con problemi. Le banche applicano elevati tassi d'interesse perche manca la concorrenza nel sistema finanziario, questo perche manca la Borsa, quindi il credito rimane l'unica risorsa di liquidità.

\footnotetext{
${ }^{4}$ Fullani , A. (2012) La conferenza stampa nel luglio 2012

${ }^{5}$ ISTAT
} 
Secondo, anche per i prestiti garantiti dal collaterale, le banche non devono affrettarsi in maniera uni laterale al pignoramento del collaterale, perche questo porterebbe al fallimento delle imprese e questa situazione sarebbe un bumerang per le banche. Un debole economia non può produrre un sano portafoglio crediti. D'altra parte questo potrebbe diventare fonte per gli abusi. Anche il nostro sistema bancario, nonostante la corruzione abbia dilagato nel giuridico e nella sanità, e il più pulito paragonato ed essi, ma nonostante ciò non e non e immune. Che cosa potrebbe accadere? Spesso le imprese potrebbero avere problemi di diversa natura, oltretutto che l'economia e entrata in una fase di crisi, e non possono restituire i debiti nei confronti delle banche. Le banche dopo che passa un anno di ritardi nel pagamento delle rate, chiede immediatamente di pignorare il collaterale vendendo quest'ultimo in aste con prezzi bassi fino al livello del debito che il cliente ha nei confronti delle banche, comprando questi collaterali persone dentro le banche o altre a loro collegate.

Questo fa si che le banche non cercano di aiutare le imprese, precisiamo per quelle imprese che possono essere aiutate, ristrutturando il loro debito, non solo nelle condizioni iniziali del contratto nei confronti della banca, come la durata, togliere le penalità, ma anche dando un prestito ulteriore, con il quale i clienti potrebbe uscire dalle difficoltà finanziarie, e potendo cosi pagare tutti i debiti che ha nei confronti della banca. Questo richiede professionalità da parte degli agenti del credito, devono conoscere molto bene le condizioni di salute dell'impresa, ed essere accorti nel fare un tale passo, ma che cosi facendo aiuterebbe sia le banche che l'economia nel futuro.

Un altro grave problema e che le banche sceglie la strada più breve, quando un impresa si trova in difficoltà, applicando interessi di mora, ma che questi ultimi danno il colpo di grazia alle imprese. A nostro avviso le banche stanno abusando nel applicare questi interessi di mora e altre multe che derivano dal ritardo del pagamento.

Terzo, quando comincerà il pignoramento di massa dei collaterali, questo può influenzare a non fare si di trovare un mercato in cui li si può vendere, il caso più negativo possibile, fenomeno realmente accaduto negli USA. Come dice il Proff. Civici", anche i possessori delle proprietà non potevano vederle da nessuna parte in maniera da liquidare i propri debiti", questo perche sceso il potere d'acquisto della popolazione. (Kriza financiare apo globale? Civici A. f. 23). L'altra parte negativa e che questo può avere un impatto nell'abbassamento dei prezzi delle proprietà immobiliari, cosa che peggiorerebbe la crisi nella quale e entrato il settore dell'edilizia ormai, quando si sa che il credito dato a questo settore raggiunge il $20 \%$ del totale prestiti dati dalla banca. In queste condizioni contribuirebbe nel fallimento da parte dei clienti delle banche. Quindi le stesse banche avrebbero problemi poiché aumenterebbe il rapporto dei prestiti con problemi.

\section{In conclusione noi suggeriamo:}

Primo: la Banca d'Albania deve prendere urgentemente dei provvedimenti per amministrare la situazione, cercando da parte di tutte le banche di presentare un pino 
d'azione per la diminuzione del livello dei prestiti con problemi, con scadenza prestabilita da parte loro e con provvedimenti concreti. Queste perche la Banca d'Albania e l'unica istituzione che può armonizzare gli interessi degli individui, economia, imprese e delle banche.

Secondo: il pignoramento del collaterale non deve farsi in blocco, ma gradualmente cercando cosi che le banche sfruttino come prima cosa la ristrutturazione del prestito, e l'esecuzione del collaterale sia l'ultima misura da intraprendere, e che sia giustificato da parte delle banche.

Terzo: Devono essere presi provvedimenti urgenti nell'abbassamento dei tassi d'interesse dei prestiti, dopo una nostra analisi, il livello dello spread tra la norma d'interesse dei prestiti e quella dei depositi e tra le più alte in confronto ai paesi della regione ( attualmente il rapporto del redito da interesse-spesa da interesse e al 137\% dicembre 2012). Positivo e il fatto che la Banca d'Albania con la pubblicazione del regolamento sulla trasparenza, ha influito in qualche maniera che il cliente potesse conoscere i reali tassi d'interesse per i prestiti che prendeva. Ma questo non basta perche non ha portato ad un loro abbassamento, questo richiederà che le banche amministrino meglio gli altri rischi con cui esse hanno a che fare, come il rischio dai tassi d'interesse come risultato della posizione aperta(GAP). Questo minimizzare le perdite derivanti da questi rischi che influiranno nell'abbassamento del costo del tasso d'interesse per i prestiti.

\section{References:}

Aisen, A.and Franken, M. (2010), "Bank Credit during the 2008 Financial Crisis: A Cross-Country Comparison” IMF Working Paper No.10 June 2010

Bank of Albania, (2012) Supervision Annual Report

Bernake, B.S. and Gertler, M. (1995) The Credit channel of monetary policy transmission", Jornal of Economic Perspectives Vol.9 (4), pp 27.48

Boyd, J. and Gianni, D. (2009) "Banking crisis and Crisis Dating: Theory and Evidence", IMF Working Paper 06/141

Carmen, R. and Rogoff, K (2008), "This Time Is Different: A Panoramic View of Eight Centuries of Financial Crises" NBER Working Paper, (March 2008)

Cani, Sh. And Sulo, H. "Sistemi Finaciar Shqiptar në Tranzicion: Progres apo Brishtësi?” Third annual Conference, Bank of Albania

Civici, A., (2009) "Globalizimi”, UET Press, Pp. 109

Civici A. (2010) Kriza financiare 2007-2009: Tronditja dhe pikëpyetjet mbi efektivitetin e kapitalizmit: Ekonomikus 5 Pp. 6

Cross-country experiences and policy implications from the global financial crisis. Economic Policy, 25: Pp 267-293.

Cappiello, L.; Kadareja, A., Sorensen, C.K. and Protopapa M. (2010) "Do bank loans and Credit Standart Affect Output? - European Central Bank Working paper No. 1150, January 2010 
Caprio, J. and Klingebiel, D. (1997). “Bank Insolvency: Bad Luck, Bad Policy or Bad Banking?” Annual World Bank Conference on Development Economies. Washington D.C. World Bank 1997 Pp 79-104

Caprio, G., Klingebiel, D., Laeven, L. and Noguera, G. (2005), "Systemic Financial Crises: Containment and Resolution. Cambridge, U.K.: Cambridge University Press.

Demianik, Y., Hermert, V. "Understanding the Subprime Mortgage Crisis” Federal Reserve Bank of St. Louis , Supervisory Policy Analysis Working Paper August 2008.

Dermirguc-Kunt, A. and Detragiache, E. (2000), "Monitoring banking sector fragility: World Bank Economic Review, 14(2), 287-307.

Driscoll, J.C. (2004), "Does bank lending affect output? Evidence from the US

Draghi M.,(2009) "Challendes to financial stability and the proposal of the Financial Stability

Bord"., 12 th Conference of the ECB-CFS Research Network, Rome, 12 November 2009.

Dungey, M., Fry, R., Gonzalez-Hermosillo, B. and Martin, V.L. (2003), "Empirical modeling of contagion: a review of methodologies", Australian National University.

Edison, H. J. (2003), "Do indicators of financial crises work?

Fullani, A. (2010) Il discorso nel seminario: "Shqipëria - mbajtja e stabilitetit makroekonomik në një ambjent të jashtëm sfidues", Hotel Tirana International, 4 Mars 2010

Gjata M. and Gjebreja E. (2010) "Risku i kredidhënies në Shqipëri”, (The Credit Risk in Albania)

Economicus 5 Pp. 146.

Hempall, H.S. and Kok Sorensen, C. (2009): “The impact of supply Constraints on Bank Lending in the Euro Area. Crisis Induced Crunching??", paper presented at the ECB Workshop (30 Nov. - 1 Dec. 2009, Frankfurt).

Huian M, 2010, "Impact of Current Financial Crisis on Disclosures of Financial Instrument"

Ignat, I. and Ifrim, M. 2010 "The current Economy Crisis and its implications on the World Economy".

IMF, (2009) "Global Financial Stability Report".

Kadia B. (2010) Politikat e Bankës së Shqipërisë gjatë krizës globale: [Bank of Albania Policies during global crisis] Economicus No.5 Pp 55.

Kaminsky, G.L., Lizondo, S. and Reinhart, C.M. (1998), "Leading indicators of currency crisis", IMF Staff Papers 45/1, International Monetary Fund, Washington, D.C.

Kaminsky, G.L. and Reinhart, C.M. (1999), "The twin crises: the causes of banking and balanceof-payments problems", American Economic Review, 89(3), Pp 473-50.

Kamin, S.B., Schindler, J.W. and Samuel, S.L. (2001), "The contribution of domestic and external sector factors to emerging market devaluations crises: an early warning systems approach", International Finance Discussions Papers 711, Board of Governors of the Federal Reserve System, Washington, D.C.

Kotorri, A. and Korbi, A. (2009) International Conference December 2009 Tirana University, Euro print ISBN: 798-99956-811-8-0 Pp 32.

Marone, H. (2009) Some effects of the financial crisis on the developing world. Office of development studies, UNDP New York.

Marchesi, S. (2003), "Adoption of an IMF program and debt rescheduling", Journal of Development Economics, 70(2), 403-42.

Nistor, I. and Ulici, M. (2009) "The financial crisis and the impact over sectors of economy", Annuals of Faculty of Economic, University of Oradea, III Pp 288-293.

Note, S. (2006) "Competition in Albanian Banking System".

Laeven and Valencia (2008)"Systemic Bank Crises: A new database "IMF working Paper 08/224.

Shkurti, R. Babasuli, J. Bahiti R. (2010), "Impact of the Latest Financial Crisis on a Bank Ba2ed Financial System -Case of Albania" The Romanian Economic Journal

Trichet, J.C. (2009) "Key lessons from the crisis" Annual conference 2009 organized by Association de Mercado's Financiers, Madrid, 23 November 2009 
Xhepa, S. (2010) "Miti mbi krizat dhe fundin e tregut" [Myth on crises and the end of market] pg.25 UET Press, 2010 ISBN 978-99956-39-31-

http://www.imf.org/external/pubs/ft/fandd/2008/06/dodd.htmhttp://www.insidemortgagefin ance.com/data/bank mortgage banking activity.html

http://press.uchicago.edu/ucp/books/book/chicago/R/bo3614501.html

http://www.sciencedirect.com/science/article/pii/S026156069900024 